\title{
Current Measurement Reliability of Selected Smoke Analytes*
}

\author{
by \\ S.W. Purkis, C.A. Hill, and I.A. Bailey \\ Imperial Tobacco Limited, P.O.Box 525, Southville, Bristol BS99 1TZ, UK.
}

\section{SUMMARY}

The reliability of measurements of mainstream smoke analytes other than "tar", nicotine and carbon monoxide $(\mathrm{CO})$ is not known but is important in the current regulatory environment internationally. An appreciation of between laboratory variability is essential for companies contracting analytical work to outside suppliers.

Seven laboratories obtained data from three cigarette brands for as many as they could currently measure of the 44 smoke analytes, commonly referred to as the "Hoffmann list". The brands, of "tar" yields $12 \mathrm{mg}, 8 \mathrm{mg}$ and $5 \mathrm{mg}$, were smoked under the International Organisation for Standardisation (ISO) smoking regime to obtain average yield values based on 5 replicates, each laboratory smoking their chosen number of cigarettes per replicate. In addition, laboratories used their preferred and internally validated methodology i.e. smoking machine type, trapping system, sample work-up and detection system. Around 3600 data points were obtained.

This study was based on one point in time measurements. It did not therefore include any components of longer-term variability that would be expected to further increase the measurement variability. No analytes had lower withinlaboratory measurement variability than "tar" and $70 \%$ of the other analytes had significantly higher levels. All laboratories ranked the products in the same order for all analytes (except some metals) but there were as much as 10 -fold differences in measured values between laboratories. The mean difference between highest and lowest yield measurements was $80 \%$ when the values for the three smoke analytes with differences in excess of 8 -fold were excluded.

Given the lack of standardised methods, and the consequent high degree of inter-laboratory variability it is not currently possible to make meaningful comparisons between such data from several sources. Indeed, calculation of yields from benchmarking studies may prove no less reliable. [Beitr. Tabakforsch. Int. 20 (2003) 314-324]

\footnotetext{
* Received: $22^{\text {nd }}$ January 2002 - accepted: $26^{\text {th }}$ June 2002

Presented in part at the CORESTA Smoke and Technology Group Meeting, Xian, China, and the Tobacco Science Research Conference Meeting, Greensboro, NC, USA, September 2001.
}

\section{ZUSAMMENFASSUNG}

Die Zuverlässigkeit der Messungen der wichtigsten Rauchanalyten des Hauptstromrauchs außer Kondensat, Nikotin und Kohlenmonoxid (CO) ist nicht bekannt, spielt jedoch im gegenwärtigen regulativen Umfeld international eine wichtige Rolle. Eine Bewertung der Messabweichungen zwischen verschiedenen Untersuchungslabors ist wichtig für Unternehmen, die derartige Analysen extern vergeben.

Sieben Untersuchungslabors ermittelten von drei Zigarettenmarken Daten so vieler Substanzen aus der Liste der 44 Rauchinhaltsstoffe, die als „Hoffmann“-Liste bekannt ist, wie es ihnen möglich war zu messen. Die Marken mit einem Kondensatgehalt von $12 \mathrm{mg}, 8 \mathrm{mg}$ und $5 \mathrm{mg}$ wurden gemäß ISO-Norm abgeraucht. Die Mittelwerte basierten auf 5 Wiederholungsmessungen, wobei jedes Untersuchungslabor die von ihm gewünschte Anzahl Zigaretten pro Messung abrauchte. Darüber hinaus benutzten die Labors ihre jeweils bevorzugte und intern validierte Methodik bezüglich Rauchmaschinentyp, Auffangsystem, Probenaufbereitung und Detektionssystem. Ungefähr 3600 Einzeldaten wurden ermittelt.

Die Studie basierte auf einem Messzeitpunkt. Aus diesem Grund ist die Studie nicht dazu angelegt, längerfristige Schwankungen zu untersuchen, von denen zu erwarten wäre, dass sie die Messabweichungen zusätzlich weiter erhöhen. Bei keinem Analyt war die laborinterne Varianz geringer als bei Kondensat, und bei $70 \%$ der anderen Analyten waren die Abweichungen signifikant erhöht. Alle Labors klassifizierten die Zigarettenmarken für alle Rauchanalyte (außer einiger Metalle) in derselben Rangfolge, dennoch variierten die Messwerte in den verschieden Labors mitunter um das Zehnfache. Die mittlere Abweichung zwischen den höchsten und niedrigesten gemessenen Werten betrug $80 \%$, wenn die Werte für drei Analyten mit mehr als 8-fachem Unterschied ausgeschlossen wurden. 
Aufgrund fehlender standardisierter Methoden und den hieraus resultierenden Abweichungen zwischen den Ergebnissen verschiedener Labors ist es gegenwärtig nicht möglich, aussagekräftige Vergleiche zwischen solchen Werten aus unterschiedlichen Quellen anzustellen. Die Berechnung von Hauptstromrauchwerten aus „Benchmarking”-Studien könnten sich als ebenso unzuverlässig erweisen. [Beitr. Tabakforsch. Int. 20 (2003) 314-324]

\section{RESUME}

La fiabilité des mesures des analytes de la fumée du courant principal autres que le goudron, la nicotine et le monoxyde de carbone $(\mathrm{CO})$ n'est pas connue, mais elle est importante à l' échelle internationale dans le contexte réglementaire actuel. L' appréciation de la variabilité des mesures entre les laboratoires est indispensable pour les sociétés confiant des travaux analytiques aux laboratoires extérieurs.

Sept laboratoires ont obtenu des données relatives à la fumée de trois marques de cigarettes pour 44 analytes de la fumée, généralement appelés "Liste Hoffmann" pour autant qu' ils pouvaient mesurer à l' époque. Les marques dont les teneurs en goudron étaient de $12 \mathrm{mg}, 8 \mathrm{mg}$ et $5 \mathrm{mg}$, ont été fumées selon la norme ISO pour obtenir des teneurs moyennes basées sur 5 répétitions, chaque laboratoire fumant un nombre choisi de cigarettes par répétition. De plus, les laboratoires ont appliqué leur méthodes préférées et validées, c' est-à-dire type de machine à fumer, système de piégeage, préparation des échantillons et système de détection. Près de 3600 données ont été recueillies.

Cette étude est basée sur des mesures uniques. Par conséquent, l' étude n' examine pas de variations à plus long terme, dont on pourrait s' attendre à une augmentation supplémentaire de l' imprécision de la mesure. Parmi tous les analytes, la variation intralaboratoire du taux de goudron s' avère la plus petite, et pour $70 \%$ des autres analytes une variation significative peut être observée. Tous les laboratoires ont classé les produits dans le même ordre pour tous les analytes (sauf certains métaux), néanmoins les valeurs mesurées varient d'un facteur 10 entre laboratoires. La différence moyenne entre les mesures des teneurs les plus et les moins élevées est de $80 \%$, lorsque 3 valeurs en excès d' un facteur de 8 sont exclues.

Etant donné l' absence de méthodes normalisées, et le degré élevé de variation entre laboratoires qui en résulte, il n' est pas possible actuellement de faire des comparaisons significatives entre de telles données provenant de plusieurs sources. En fait, le calcul des teneurs provenant d'études de référence pourrait s' avérer non moins fiable. [Beitr. Tabakforsch. Int. 20 (2003) 314-324]

\section{INTRODUCTION}

The reliability of measurements of mainstream smoke analytes other than "tar", nicotine and carbon monoxide (CO) is not known but is important in the current international regulatory environment. An appreciation of between laboratory variability is essential for companies involved in selecting laboratories for measurements or in comparing product data gathered from different laboratories.
Previous "benchmarking" studies have coped with between laboratory variability either by distributing analyte measurements so that particular laboratories measure particular analytes (1), or by using one laboratory for all analyte measurements, irrespective of the representative nature or quality of the data it generates (2).

Very few publications are available giving inter-laboratory comparisons although HsU et al. recently reported a series of cigarette analyses and method validation (3). They concluded that for these laboratories adopting their own protocols, differences of greater than $30 \%$ were found in smoke yields.

The present study compares smoke data generated at different laboratories when testing cigarettes from a common sample. It measures the inter-laboratory variability between up to seven laboratories for a series of three Imperial Tobacco Limited cigarette products designed at three different "tar" levels and containing mainly flue-cured tobaccos.

It should be noted that the study design eliminated both longer-term product and testing variability, both of which would be expected to increase the overall variability in these measurements.

\section{METHODS}

Three cigarette products produced during July or August 2000 were supplied to the laboratories for the smoke analyses, which were carried out between September 2000 and July 2001. These cigarette products contained essentially flue-cured tobacco and their designs covered a range of "tar" values i.e. $12 \mathrm{mg}$ (Product A), $8 \mathrm{mg}$ (Product B) and $5 \mathrm{mg}$ (Product C). Matched samples were used in this work to reduce product variability to a minimum.

Two non-tobacco industry contract laboratories and two tobacco manufacturers' laboratories carried out extensive smoke analyses on the 44 smoke analytes commonly referred to as the "Hoffmann list". In addition, another tobacco manufacturer's laboratory and two non-tobacco industry laboratories were able to measure some of the selected smoke analytes at the time of the study.

Currently there are no internationally recognised standard methods for these analytes, apart from "tar", nicotine and carbon monoxide (CO). For each smoke analyte, each laboratory used whichever smoking machines (linear or rotary), trapping systems and analytical methodology their experts considered best and used as part of their normal practice. Each laboratory also applied their internal validation process. A summary of the analytical methods employed at each laboratory and the number of cigarettes smoked per determination for the different methods is given in Table 1 . It was recognised that this selection method would better reflect the current state of analytical expertise despite potentially increasing variability. Some recognised sources of variability are the smoking machine type and set-up, smoke trapping efficiencies, puff profile changes when trapping into liquid traps, sample work-up, instrument measurements, interference from other smoke components and calibration standards.

Cigarettes were conditioned in compliance with ISO 3402 (4) and smoked following the requirements of ISO 3308 (5) 


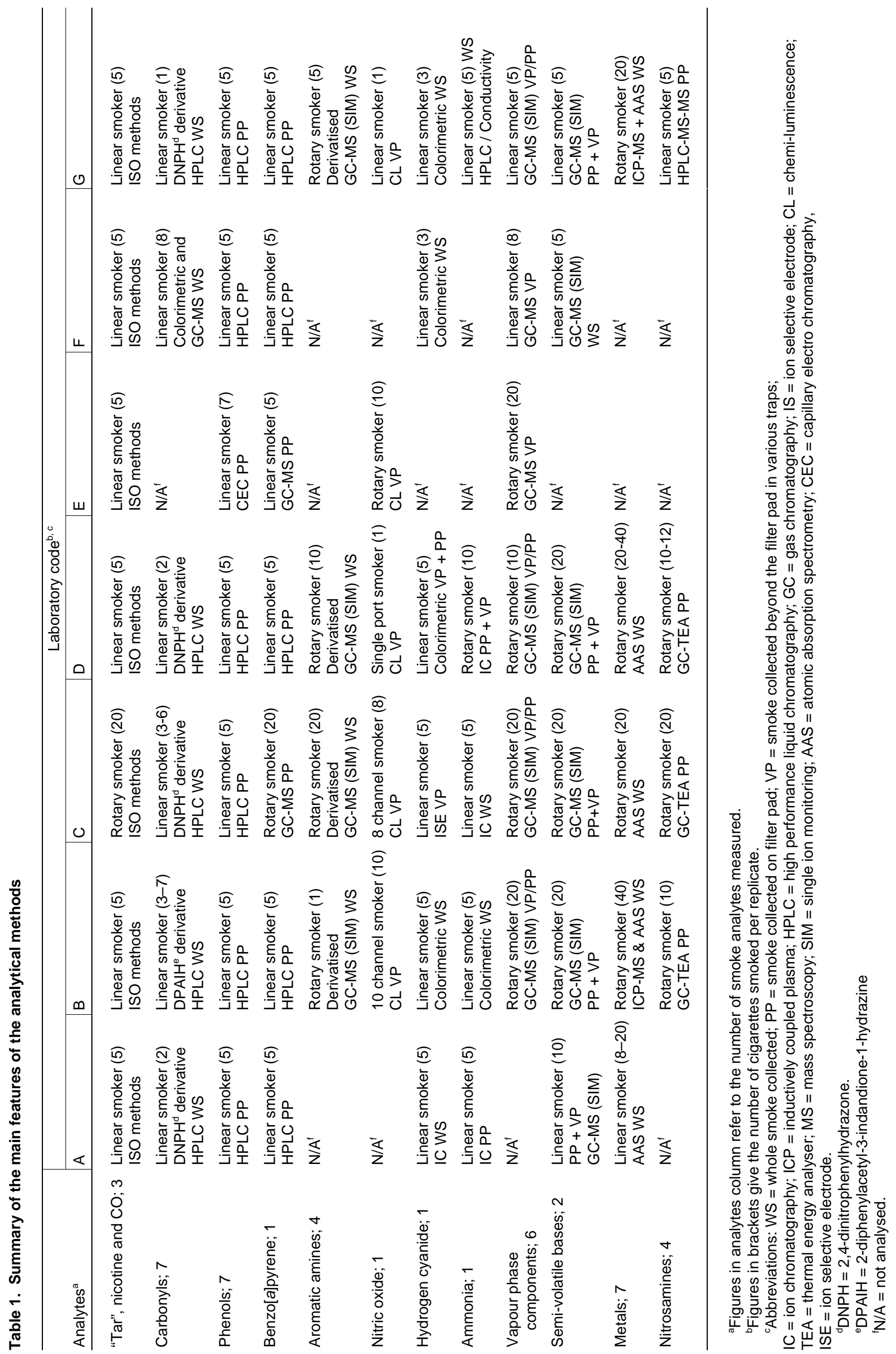


Table 2. Smoke analyte mean smoke yields - Product A

\begin{tabular}{|c|c|c|c|c|c|c|c|c|c|}
\hline \multirow[b]{2}{*}{ Analytes } & \multirow[b]{2}{*}{ Units } & \multicolumn{7}{|c|}{ Laboratory code } & \multirow[b]{2}{*}{ Mean } \\
\hline & & A & $\mathrm{B}$ & $\mathrm{C}$ & $\mathrm{D}$ & $E$ & $\mathrm{~F}$ & $\mathrm{G}$ & \\
\hline NFDPM $^{a}$ & $\mathrm{mg} / \mathrm{cig}$ & 12.4 & 12.0 & 12.4 & 12.6 & 12.2 & 12.7 & 14.0 & 12.6 \\
\hline Nicotine & $\mathrm{mg} / \mathrm{cig}$ & 1.03 & 0.98 & 1.20 & 1.05 & 1.00 & 1.03 & 0.98 & 1.04 \\
\hline Carbon monoxide & $\mathrm{mg} / \mathrm{cig}$ & 14.2 & 13.6 & 16.2 & 14.0 & 15.3 & 13.3 & 15.6 & 14.6 \\
\hline Benzo[a]pyrene & ng/cig & 11.1 & 11.6 & 9.8 & 14.8 & 15.5 & 9.6 & 13.2 & 11.4 \\
\hline Nitric oxide & $\mu \mathrm{g} / \mathrm{cig}$ & - & 128.3 & 127.8 & 136.3 & 122.6 & - & 176.4 & 138.3 \\
\hline Hydrogen cyanide & $\mu \mathrm{g} / \mathrm{cig}$ & 160 & 170 & 124 & 149 & - & 183 & 143 & 157 \\
\hline Ammonia & $\mu \mathrm{g} / \mathrm{cig}$ & 6.2 & 16.6 & 11.1 & 12.9 & - & - & 7.5 & 11.7 \\
\hline Benzene & $\mu \mathrm{g} / \mathrm{cig}$ & - & 54.5 & 51.6 & 45.3 & 44.1 & 60.8 & 64.5 & 53.5 \\
\hline Toluene & $\mu \mathrm{g} / \mathrm{cig}$ & - & 72.1 & 83.2 & 67.0 & 65.3 & 68.6 & 106.4 & 77.1 \\
\hline Styrene & $\mu \mathrm{g} / \mathrm{cig}$ & - & 6.0 & 23.8 & 9.8 & 2.4 & - & 13.2 & 11.1 \\
\hline 1,3-Butadiene & $\mu \mathrm{g} / \mathrm{cig}$ & - & 76.5 & 21.9 & 50.7 & - & - & 40.3 & 47.4 \\
\hline Isoprene & $\mu \mathrm{g} / \mathrm{cig}$ & - & 470 & 394 & 337 & 364 & 489 & 352 & 401 \\
\hline Acrylonitrile & $\mu \mathrm{g} / \mathrm{cig}$ & - & 13.4 & 16.2 & 9.0 & 12.2 & 15.0 & 13.5 & 13.9 \\
\hline Quinoline & $\mu g / c i g$ & 0.5 & 0.5 & 0.4 & 0.4 & - & 1.4 & 0.3 & 0.6 \\
\hline Pyridine & $\mu g / c i g$ & 11.1 & 5.5 & 11.8 & 12.1 & - & 10.4 & 9.2 & 10.1 \\
\hline Phenol & $\mu g /$ cig & 16.5 & 16.2 & 18.4 & 18.9 & 38.1 & 19.3 & 18.0 & 20.8 \\
\hline$m / p$-Cresol & $\mu \mathrm{g} / \mathrm{cig}$ & 8.3 & 11.0 & 10.8 & 12.8 & 8.7 & 6.4 & 10.9 & 9.8 \\
\hline o-Cresol & $\mu \mathrm{g} / \mathrm{cig}$ & 7.4 & 3.9 & 4.2 & 4.9 & 2.8 & 5.4 & 4.6 & 4.7 \\
\hline Formaldehyde & $\mu \mathrm{g} / \mathrm{cig}$ & 67.5 & 76.5 & 72.6 & 59.6 & - & 61.8 & 74.4 & 68.7 \\
\hline Acetaldehyde & $\mu \mathrm{g} / \mathrm{cig}$ & 843 & 1111 & 818 & 792 & - & 1036 & 910 & 918 \\
\hline Acetone & $\mu g / c i g$ & 377 & 362 & 368 & 394 & - & 373 & 434 & 385 \\
\hline 2-Butanone & $\mu \mathrm{g} / \mathrm{cig}$ & - & 102.8 & 115.4 & 76.4 & - & 94.2 & 168.4 & 111.5 \\
\hline Propanal & $\mu \mathrm{g} / \mathrm{cig}$ & 60.4 & 67.9 & 68.2 & 70.9 & - & 50.2 & 82.1 & 66.6 \\
\hline Butanal & $\mu \mathrm{g} / \mathrm{cig}$ & 29.2 & - & 61.0 & 39.8 & - & 48.8 & 48.6 & 45.5 \\
\hline Crotonaldehyde & $\mu \mathrm{g} / \mathrm{cig}$ & 35.4 & 33.9 & 25.7 & 23.0 & - & 23.0 & 45.0 & 31.0 \\
\hline $\mathrm{NNK}^{\mathrm{b}}$ & $\mathrm{ng} / \mathrm{cig}$ & - & 41.5 & 35.0 & 31.6 & - & - & 31.6 & 34.9 \\
\hline$N$-Nitrosonornicotine & $\mathrm{ng} / \mathrm{cig}$ & - & 22.8 & 16.3 & 24.2 & - & - & 20.0 & 21.1 \\
\hline N-Nitrosoanatabine & $\mathrm{ng} / \mathrm{cig}$ & - & 45.6 & 34.7 & 34.5 & - & - & 26.9 & 38.3 \\
\hline$N$-Nitrosoanabasine & $\mathrm{ng} / \mathrm{cig}$ & - & 8.3 & $<7$ & 3.7 & - & - & 5.1 & 6.0 \\
\hline 4-Aminobiphenyl & $\mathrm{ng} / \mathrm{cig}$ & - & 1.0 & 1.3 & 1.8 & - & - & 1.3 & 1.4 \\
\hline 3-Aminobiphenyl & $\mathrm{ng} / \mathrm{cig}$ & - & 1.5 & 1.6 & 2.5 & - & - & 1.6 & 1.8 \\
\hline 2-Naphthylamine & $\mathrm{ng} / \mathrm{cig}$ & - & 3.9 & 8.1 & 8.5 & - & - & 7.5 & 7.0 \\
\hline 1-Naphthylamine & $\mathrm{ng} / \mathrm{cig}$ & - & 11.9 & 12.0 & 14.1 & - & - & 11.7 & 12.4 \\
\hline Resorcinol & $\mu \mathrm{g} / \mathrm{cig}$ & 1.2 & 1.3 & 1.3 & 0.7 & 6.7 & 8.1 & 1.4 & 3.0 \\
\hline Hydroquinone & $\mu \mathrm{g} / \mathrm{cig}$ & 72.2 & 75.0 & 81.7 & 73.7 & 68.4 & 83.8 & 71.3 & 75.1 \\
\hline Catechol & $\mu \mathrm{g} / \mathrm{cig}$ & 74.3 & 76.5 & 83.1 & 71.5 & 60.5 & 56.3 & 78.9 & 71.6 \\
\hline Chromium & $\mathrm{ng} / \mathrm{cig}$ & 12.4 & $<5$ & $<2$ & 5.0 & - & - & 2.5 & 6.6 \\
\hline Cadmium & $\mathrm{ng} / \mathrm{cig}$ & 23.3 & 23.7 & 40.2 & 36.8 & - & - & 29.5 & 30.7 \\
\hline Lead & $\mathrm{ng} / \mathrm{cig}$ & 22.1 & 15.5 & 12.0 & 29.2 & - & - & 18.3 & 19.4 \\
\hline Mercury & $\mathrm{ng} / \mathrm{cig}$ & 3.4 & 2.0 & 0.4 & 4.1 & - & - & - & 2.5 \\
\hline Nickel & ng/cig & $<2$ & $<6$ & $<3$ & 4.6 & - & - & $<2.1$ & 4.6 \\
\hline Selenium & ng/cig & $<2$ & $<6$ & $<1.3$ & 1.1 & - & - & $<0.9$ & 1.1 \\
\hline Arsenic & ng/cig & 2.2 & 1.7 & 1.1 & 1.6 & - & - & 1.8 & 1.7 \\
\hline Acrolein & $\mu \mathrm{g} / \mathrm{cig}$ & - & 102.9 & 48.7 & 85.9 & - & 68.8 & 105.2 & 76.6 \\
\hline "Smoke pH" & & 6.0 & 6.1 & 5.0 & - & - & 5.1 & - & 5.5 \\
\hline
\end{tabular}

aNFDPM = nicotine free dry particulate matter ("tar").

${ }^{b} \mathrm{NNK}=4-(N$-methyl- $N$-nitrosamino)-1-(3-pyridyl)-1-butanone.

as closely as possible. Five replicates were made per analyte. Around 3600 data points were collected during this work. On external statistical advice, four outliers were excluded from the analyses in line with ISO 5725-2 guidelines (6).

This study was based on one point in time measurements and did not include any components of longer-term variability.

\section{DISCUSSION OF RESULTS}

A summary of the mean values obtained by each laboratory for each brand is given in Tables 2 to 4 .

\section{Puff number}

One parameter that might be expected to directly influence the yield of all analytes is the puff number (PN). In addi- 
Table 3. Smoke analyte mean smoke yields - Product B

\begin{tabular}{|c|c|c|c|c|c|c|c|c|c|}
\hline \multirow[b]{2}{*}{ Analytes } & \multirow[b]{2}{*}{ Units } & \multicolumn{7}{|c|}{ Laboratory code } & \multirow[b]{2}{*}{ Mean } \\
\hline & & $\mathrm{A}$ & $\mathrm{B}$ & $\mathrm{C}$ & $\mathrm{D}$ & $E$ & $\mathrm{~F}$ & $\mathrm{G}$ & \\
\hline NFDPM $^{a}$ & $\mathrm{mg} / \mathrm{cig}$ & 7.1 & 6.8 & 7.3 & 7.5 & 7.4 & 7.5 & 8.8 & 7.5 \\
\hline Nicotine & $\mathrm{mg} / \mathrm{cig}$ & 0.71 & 0.70 & 0.87 & 0.74 & 0.69 & 0.70 & 0.72 & 0.73 \\
\hline Carbon monoxide & $\mathrm{mg} / \mathrm{cig}$ & 6.8 & 6.3 & 7.6 & 7.0 & 7.4 & 6.3 & 8.0 & 7.1 \\
\hline Benzo[a]pyrene & ng/cig & 5.1 & 5.7 & 4.4 & 7.6 & 8.4 & 5.0 & 7.0 & 5.6 \\
\hline Nitric oxide & $\mu \mathrm{g} / \mathrm{cig}$ & - & 56.5 & 56.0 & 65.0 & 58.2 & - & 84.9 & 64.1 \\
\hline Hydrogen cyanide & $\mu \mathrm{g} / \mathrm{cig}$ & 68 & 89 & 62 & 65 & - & 134 & 90 & 84 \\
\hline Ammonia & $\mu \mathrm{g} / \mathrm{cig}$ & 4.2 & 10.6 & 7.4 & 8.6 & - & - & 5.4 & 7.7 \\
\hline Benzene & $\mu g /$ cig & - & 28.5 & 25.5 & 23.3 & 21.8 & 45.8 & 37.9 & 30.5 \\
\hline Toluene & $\mu g /$ cig & - & 38.3 & 45.8 & 34.6 & 32.7 & 52.2 & 59.9 & 43.9 \\
\hline Styrene & $\mu \mathrm{g} / \mathrm{cig}$ & - & 2.8 & 10.3 & 4.8 & 1.3 & - & 7.0 & 5.2 \\
\hline 1,3-Butadiene & $\mu \mathrm{g} / \mathrm{cig}$ & - & 34.9 & 15.1 & 29.6 & - & - & 25.8 & 26.4 \\
\hline Isoprene & $\mu \mathrm{g} / \mathrm{cig}$ & - & 276 & 322 & 235 & 191 & 460 & 281 & 294 \\
\hline Acrylonitrile & $\mu \mathrm{g} / \mathrm{cig}$ & - & 6.4 & 8.2 & 8.2 & 4.4 & 11.6 & 7.3 & 7.7 \\
\hline Quinoline & $\mu g / c i g$ & 0.4 & 0.3 & 0.3 & 0.3 & - & 1.1 & 0.3 & 0.5 \\
\hline Pyridine & $\mu \mathrm{g} / \mathrm{cig}$ & 7.0 & 3.5 & 6.4 & 6.4 & - & 8.3 & 6.0 & 6.3 \\
\hline Phenol & $\mu \mathrm{g} / \mathrm{cig}$ & 14.1 & 14.3 & 17.3 & 13.4 & 34.1 & 15.3 & 14.5 & 17.6 \\
\hline$m / p$-Cresol & $\mu \mathrm{g} / \mathrm{cig}$ & 6.9 & 9.4 & 9.7 & 9.1 & 6.7 & 5.3 & 8.6 & 8.0 \\
\hline o-Cresol & $\mu \mathrm{g} / \mathrm{cig}$ & 5.2 & 3.5 & 3.5 & 3.5 & 2.8 & 4.3 & 3.6 & 3.8 \\
\hline Formaldehyde & $\mu \mathrm{g} / \mathrm{cig}$ & 34.9 & 35.8 & 35.6 & 30.1 & - & 40.8 & 38.1 & 35.9 \\
\hline Acetaldehyde & $\mu \mathrm{g} / \mathrm{cig}$ & 466 & 571 & 419 & 414 & - & 738 & 521 & 518 \\
\hline Acetone & $\mu \mathrm{g} / \mathrm{cig}$ & 231 & 192 & 186 & 214 & - & 282 & 272 & 229 \\
\hline 2-Butanone & $\mu \mathrm{g} / \mathrm{cig}$ & - & 59.3 & 58.9 & 43.2 & - & 71.0 & 95.8 & 65.6 \\
\hline Propanal & $\mu g / c i g$ & 34.1 & 36.7 & 35.3 & 37.9 & - & 37.8 & 48.8 & 38.4 \\
\hline Butanal & $\mu \mathrm{g} / \mathrm{cig}$ & 17.3 & - & 38.6 & 24.6 & - & 35.2 & 29.2 & 29.0 \\
\hline Crotonaldehyde & $\mu g / c i g$ & 17.8 & 15.5 & 13.5 & 10.0 & - & 18.4 & 25.1 & 16.8 \\
\hline$N_{N K}^{b}$ & $\mathrm{ng} / \mathrm{cig}$ & - & 30.3 & 29.0 & 29.0 & - & - & 24.8 & 28.3 \\
\hline$N$-Nitrosonornicotine & $\mathrm{ng} / \mathrm{cig}$ & - & 19.1 & 15.4 & 21.1 & - & - & 17.1 & 18.5 \\
\hline $\mathrm{N}$-Nitrosoanatabine & $\mathrm{ng} / \mathrm{cig}$ & - & 36.6 & 32.0 & 30.3 & - & - & 26.1 & 33.0 \\
\hline $\mathrm{N}$-Nitrosoanabasine & ng/cig & - & 7.1 & $<7$ & 3.3 & - & - & 3.9 & 5.2 \\
\hline 4-Aminobiphenyl & $\mathrm{ng} / \mathrm{cig}$ & - & 0.7 & 1.0 & 1.3 & - & - & 1.0 & 1.0 \\
\hline 3-Aminobiphenyl & $\mathrm{ng} / \mathrm{cig}$ & - & 1.1 & 1.1 & 1.8 & - & - & 1.3 & 1.3 \\
\hline 2-Naphthylamine & $\mathrm{ng} / \mathrm{cig}$ & - & 2.9 & 5.4 & 6.1 & - & - & 6.0 & 5.1 \\
\hline 1-Naphthylamine & $\mathrm{ng} / \mathrm{cig}$ & - & 7.9 & 8.4 & 9.7 & - & - & 8.4 & 8.6 \\
\hline Resorcinol & $\mu g / c i g$ & 0.7 & 0.9 & 0.8 & 0.6 & 3.1 & 5.6 & 1.0 & 1.8 \\
\hline Hydroquinone & $\mu \mathrm{g} / \mathrm{cig}$ & 42.9 & 49.2 & 55.1 & 43.6 & 46.7 & 70.9 & 47.5 & 50.8 \\
\hline Catechol & $\mu \mathrm{g} / \mathrm{cig}$ & 43.3 & 48.0 & 55.1 & 41.4 & 38.9 & 35.2 & 49.7 & 44.5 \\
\hline Chromium & $\mathrm{ng} / \mathrm{cig}$ & 8.7 & $<5$ & $<2$ & 4.7 & - & - & 1.9 & 5.1 \\
\hline Cadmium & $\mathrm{ng} / \mathrm{cig}$ & 20.6 & 22.2 & 28.2 & 35.3 & - & - & 29.4 & 27.1 \\
\hline Lead & ng/cig & 11.4 & 8.8 & 10.4 & 16.8 & - & - & 9.4 & 11.3 \\
\hline Mercury & ng/cig & 3.5 & 1.5 & 0.4 & 3.1 & - & - & - & 2.1 \\
\hline Nickel & ng/cig & $<2$ & $<6$ & $<3$ & 5.3 & - & - & $<2.1$ & 5.3 \\
\hline Selenium & $\mathrm{ng} / \mathrm{cig}$ & $<2$ & $<6$ & $<1.3$ & 0.8 & - & - & $<0.9$ & 0.8 \\
\hline Arsenic & $\mathrm{ng} / \mathrm{cig}$ & 2.2 & 2.1 & 1.9 & 1.9 & - & - & 2.0 & 2.0 \\
\hline Acrolein & $\mu \mathrm{g} / \mathrm{cig}$ & 49.4 & 46.8 & 41.7 & 42.1 & - & 49.4 & 54.0 & 47.2 \\
\hline "Smoke pH" & & 6.3 & 6.3 & 5.1 & - & - & 5.4 & - & 5.8 \\
\hline
\end{tabular}

aNFDPM = nicotine free dry particulate matter ("tar").

${ }^{b} N N K=4-(N$-methyl- $N$-nitrosamino)-1-(3-pyridyl)-1-butanone.

tion, since it is not possible to set the airflow specified in ISO 3308 (5) with some of the trapping systems the PN would be expected to vary more than for normal ISO smoking conditions. Table 5 shows that there were differences in PN of one whole puff within one brand between laboratories for the different groups of analytes. However, the laboratory with the highest PN on one brand did not always give the highest PN on the other brands. High PNs did not necessarily mean that a high yield was obtained. For example, vapour-phase compounds analysed by laboratory
D had the highest PN, but gave amongst the lowest yields. Within this data set, it was observed that the effect of PN on yield was likely to be relatively small compared with some of the large yield differences observed between laboratories.

\section{Repeatability (within laboratory variability)}

Repeatability $(r)$ is reflected in the mean coefficient of variation $(\mathrm{CoV})$ values for each analyte across all products 
Table 4. Smoke analyte mean smoke yields - Product C

\begin{tabular}{|c|c|c|c|c|c|c|c|c|c|}
\hline \multirow[b]{2}{*}{ Analytes } & \multirow[b]{2}{*}{ Units } & \multicolumn{7}{|c|}{ Laboratory code } & \multirow[b]{2}{*}{ Mean } \\
\hline & & $\mathrm{A}$ & $\mathrm{B}$ & $\mathrm{C}$ & $\mathrm{D}$ & $\mathrm{E}$ & $\mathrm{F}$ & $\mathrm{G}$ & \\
\hline NFDPM $^{\mathrm{a}}$ & $\mathrm{mg} / \mathrm{cig}$ & 5.0 & 4.9 & 5.3 & 5.4 & 5.0 & 5.6 & 6.0 & 5.3 \\
\hline Nicotine & $\mathrm{mg} / \mathrm{cig}$ & 0.52 & 0.52 & 0.62 & 0.54 & 0.51 & 0.53 & 0.54 & 0.54 \\
\hline Carbon monoxide & $\mathrm{mg} / \mathrm{cig}$ & 5.7 & 5.6 & 6.7 & 6.1 & 6.6 & 5.8 & 6.6 & 6.1 \\
\hline Benzo[a]pyrene & ng/cig & 4.5 & 4.8 & 3.6 & 7.0 & 7.6 & 4.1 & 7.0 & 5.0 \\
\hline Nitric oxide & $\mu g /$ cig & - & 59.5 & 58.5 & 56.7 & 53.5 & - & 85.6 & 62.7 \\
\hline Hydrogen cyanide & $\mu \mathrm{g} / \mathrm{cig}$ & 58 & 55 & 53 & 47 & - & 106 & 90 & 64 \\
\hline Ammonia & $\mu \mathrm{g} / \mathrm{cig}$ & 3.0 & 7.2 & 5.1 & 6.4 & - & - & 3.9 & 5.4 \\
\hline Benzene & $\mu \mathrm{g} / \mathrm{cig}$ & - & 25.9 & 24.8 & 20.7 & 22.2 & 38.2 & 31.4 & 27.2 \\
\hline Toluene & $\mu \mathrm{g} / \mathrm{cig}$ & - & 33.3 & 43.3 & 30.1 & 31.7 & 32.0 & 47.9 & 36.4 \\
\hline Styrene & $\mu \mathrm{g} / \mathrm{cig}$ & - & 1.7 & 7.8 & 3.8 & 1.2 & - & 4.4 & 3.8 \\
\hline 1,3-Butadiene & $\mu \mathrm{g} / \mathrm{cig}$ & - & 34.6 & 11.3 & 25.4 & - & - & 20.9 & 23.1 \\
\hline Isoprene & $\mu \mathrm{g} / \mathrm{cig}$ & - & 260 & 265 & 198 & 203 & 361 & 215 & 250 \\
\hline Acrylonitrile & $\mu \mathrm{g} / \mathrm{cig}$ & - & 5.6 & 6.5 & 3.5 & 5.9 & 8.8 & 5.5 & 6.1 \\
\hline Quinoline & $\mu \mathrm{g} / \mathrm{cig}$ & 0.3 & 0.2 & 0.2 & 0.2 & - & 0.4 & 0.2 & 0.2 \\
\hline Pyridine & $\mu \mathrm{g} / \mathrm{cig}$ & 3.2 & 2.2 & 4.4 & 4.4 & - & 2.4 & 3.5 & 3.4 \\
\hline Phenol & $\mu \mathrm{g} / \mathrm{cig}$ & 8.6 & 9.2 & 9.9 & 9.5 & 22.3 & 9.8 & 10.3 & 11.4 \\
\hline$m / p$-Cresol & $\mu \mathrm{g} / \mathrm{cig}$ & 4.4 & 6.5 & 5.9 & 6.9 & 4.5 & 3.8 & 6.3 & 5.4 \\
\hline o-Cresol & $\mu \mathrm{g} / \mathrm{cig}$ & 3.7 & 2.4 & 2.2 & 2.7 & $<2$ & 3.1 & 2.7 & 2.8 \\
\hline Formaldehyde & $\mu \mathrm{g} / \mathrm{cig}$ & 23.6 & 22.4 & 27.1 & 17.0 & - & 31.8 & 17.8 & 23.3 \\
\hline Acetaldehyde & $\mu \mathrm{g} / \mathrm{cig}$ & 433 & 507 & 375 & 367 & - & 630 & 428 & 457 \\
\hline Acetone & $\mu \mathrm{g} / \mathrm{cig}$ & 231 & 172 & 186 & 201 & - & 247 & 230 & 211 \\
\hline 2-Butanone & $\mu \mathrm{g} / \mathrm{cig}$ & - & 54.1 & 54.5 & 37.1 & - & 61.2 & 81.6 & 57.7 \\
\hline Propanal & $\mu \mathrm{g} / \mathrm{cig}$ & 32.8 & 32.3 & 31.9 & 34.0 & - & 32.6 & 39.9 & 33.9 \\
\hline Butanal & $\mu \mathrm{g} / \mathrm{cig}$ & 17.1 & - & 35.9 & 19.1 & - & 32.6 & 24.8 & 25.9 \\
\hline Crotonaldehyde & $\mu \mathrm{g} / \mathrm{cig}$ & 17.6 & 11.9 & 11.1 & 8.6 & - & 14.0 & 19.9 & 13.9 \\
\hline$N_{N K}^{b}$ & $\mathrm{ng} / \mathrm{cig}$ & - & 19.2 & 13.6 & 16.3 & - & - & 16.9 & 16.4 \\
\hline$N$-Nitrosonornicotine & $\mathrm{ng} / \mathrm{cig}$ & - & 12.2 & 7.1 & 17.4 & - & - & 11.1 & 12.3 \\
\hline$N$-Nitrosoanatabine & $\mathrm{ng} / \mathrm{cig}$ & - & 22.4 & 17.0 & 20.1 & - & - & 19.4 & 19.9 \\
\hline$N$-Nitrosoanabasine & ng/cig & - & 5.8 & $<7$ & 3.1 & - & - & 2.8 & 4.4 \\
\hline 4-Aminobiphenyl & $\mathrm{ng} / \mathrm{cig}$ & - & 0.6 & 0.7 & 1.1 & - & - & 0.9 & 0.8 \\
\hline 3-Aminobiphenyl & $\mathrm{ng} / \mathrm{cig}$ & - & 1.1 & 0.9 & 1.4 & - & - & 1.0 & 1.1 \\
\hline 2-Naphthylamine & $\mathrm{ng} / \mathrm{cig}$ & - & 2.7 & 4.6 & 5.0 & - & - & 5.2 & 4.4 \\
\hline 1-Naphthylamine & $\mathrm{ng} / \mathrm{cig}$ & - & 6.8 & 6.7 & 8.1 & - & - & 7.2 & 7.2 \\
\hline Resorcinol & $\mu \mathrm{g} / \mathrm{cig}$ & 0.6 & 0.6 & 0.6 & 0.4 & 2.1 & 3.2 & 0.7 & 1.2 \\
\hline Hydroquinone & $\mu \mathrm{g} / \mathrm{cig}$ & 35.3 & 36.9 & 40.5 & 34.5 & 33.1 & 61.2 & 36.2 & 39.7 \\
\hline Catechol & $\mu \mathrm{g} / \mathrm{cig}$ & 37.3 & 38.2 & 43.5 & 35.0 & 33.4 & 29.1 & 41.4 & 36.9 \\
\hline Chromium & $\mathrm{ng} / \mathrm{cig}$ & 5.7 & $<5$ & $<2$ & 4.8 & - & - & $<1.7$ & 5.3 \\
\hline Cadmium & $\mathrm{ng} / \mathrm{cig}$ & 6.4 & 6.7 & 10.2 & 13.3 & - & - & 10.2 & 9.4 \\
\hline Lead & $\mathrm{ng} / \mathrm{cig}$ & 7.1 & 7.0 & 12.0 & 22.6 & - & - & 7.7 & 11.3 \\
\hline Mercury & $\mathrm{ng} / \mathrm{cig}$ & 5.0 & 1.8 & 0.4 & 2.7 & - & - & - & 2.5 \\
\hline Nickel & $\mathrm{ng} / \mathrm{cig}$ & $<2$ & $<6$ & $<3$ & 5.2 & - & - & $<2.1$ & 5.2 \\
\hline Selenium & $\mathrm{ng} / \mathrm{cig}$ & $<2$ & $<6$ & $<1.3$ & 1.0 & - & - & $<0.9$ & 1.0 \\
\hline Arsenic & $\mathrm{ng} / \mathrm{cig}$ & 1.1 & 0.8 & $<0.7$ & 0.8 & - & - & $<1$ & 0.9 \\
\hline Acrolein & $\mu g / c i g$ & 45.1 & 38.4 & 30.1 & 36.6 & - & 36.8 & 41.8 & 38.1 \\
\hline "Smoke pH" & & 7.0 & 6.3 & 5.1 & - & - & 5.8 & - & 6.0 \\
\hline
\end{tabular}

${ }^{a}$ NFDPM = nicotine free dry particulate matter ("tar").

${ }^{b} \mathrm{NNK}=4-(N$-methyl- $N$-nitrosamino $)$-1-(3-pyridyl)-1-butanone.

and for all laboratories (i.e. the full available data set). Yields of four metals (arsenic, selenium, chromium and nickel) were consistently below the quantification limit and were excluded from all the following analyses. The other analytes have been ranked in ascending order of $\mathrm{CoV}$ values in Figure 1. Twenty-eight of the 40 analytes studied have a significantly higher variation than "tar" (at the $1 \%$ level of significance) as shown by a CoV greater than or equal to $7 \%$. With one or two exceptions, there appeared to be no common factors (e.g. methodology and chemical class) making some analytes more or less variable than others. The average $\mathrm{CoV}$ for all the analyses carried out on the three brands for each laboratory was similar and ranged from $6.4-9.0 \%$ except for one laboratory where certain analytes gave much higher variability and increased their mean $\mathrm{CoV}$ to $14.3 \%$.

It is recognised that "tar", nicotine and carbon monoxide determined yields are more variable for low "tar" products and this is supported in ISO 8243 (7) by the higher tolerance (the greater of $\pm 1 \mathrm{mg}$ or $\pm 20 \%$ ) for low "tar" products 
Table 5. Comparison of mean puff numbers obtained in different analyses ${ }^{\mathrm{a}}$

\begin{tabular}{l|c|c|c}
\hline \multirow{2}{*}{ Analytes } & \multicolumn{3}{|c}{ Cigarette product } \\
\cline { 2 - 4 } "Tar", nicotine, CO & $\mathrm{A}$ & $\mathrm{B}$ & $\mathrm{C}$ \\
Metals & 8.3 & 7.2 & 7.0 \\
Phenols & 8.4 & 7.3 & 7.1 \\
Tobacco-specific nitrosamines & 8.1 & 7.2 & 6.8 \\
Carbonyls & 8.6 & 7.5 & 7.4 \\
Amines & 8.2 & 7.3 & 6.9 \\
Selected vapour-phase & 8.4 & 7.4 & 7.2 \\
$\quad$ components & 8.3 & 7.3 & 7.0 \\
Ammonia & & & \\
Hydrogen cyanide & 8.4 & 7.2 & 7.0 \\
Nitric oxide & 8.2 & 7.1 & 6.9 \\
Bases & 8.5 & 7.5 & 7.1 \\
Benzo[a]pyrene & 8.2 & 7.2 & 7.0 \\
\end{tabular}

${ }^{a}$ These values represent the mean puffs obtained across all replicates and across all laboratories.

i.e. the lower the "tar" yield the more that it varies. The differences in CoVs between the higher and lower "tar" products are shown in Figure 2. For 31 of the studied 40 analytes, higher $\mathrm{CoV}$ values were obtained for the lower "tar" product.

\section{Reproducibility (inter-laboratory variability)}

A full statistical test of reproducibility $(R)$ and repeatability $(r)$ would not be meaningful as there is insufficient data and an unbalanced design in this study. However, a less rigorous analysis is available.

For a one point in time "tar" measurement based on 20 replicates, the cigarette sampling method, ISO 8243 (7), sets a $95 \%$ confidence limit of $20 \%$ for duplicate measurements at a different laboratory corresponding to a possible ratio of 1.2 . In this work only 5 replicates were made but the average of 1.21 is not inconsistent with the ISO tolerance.

The ratio of the highest to the lowest yield value between laboratories averaged for the three brands is given in Figure 3. For most analytes these ratios are much higher than for "tar", nicotine and CO and would suggest that higher tolerances would be appropriate for these analytes. The analytes with the highest ratios are generally those that prove the most challenging to the analyst because of their chemical instability or their presence at very low levels where the measurement error will be proportionately greater.

The mean variation between highest and lowest yield measurements was $80 \%$ even after excluding 3 values in excess of 8-fold (mercury, resorcinol and styrene). The median variation was $74 \%$ for the full 40 -analyte data set. There are few obvious links between the levels of variability and the analyte chemistry.

This work suggests that, on average, yield differences measured in different laboratories on analytes other than "tar", nicotine and carbon monoxide need to be greater than $80 \%$ in order to be confident of a real difference between products.

\section{Correlation of "tar" and CO with analytes}

The mean analyte yields for each brand across all laboratories were plotted against either mean "tar" or CO yields, depending on whether the analyte was associated with the particulate or vapour phase (as indicated in Figures 4 and 5 respectively).

Although there is inter-laboratory variability for each analyte for the three products, within-laboratory comparisons ranked the analyte yields of the three products consistently relative to "tar" or CO yields. Therefore, it was considered valid to combine data from all the laboratories to give the overall lines of correlation with "tar" and CO. For 3 data points, an $R^{2}$ value of 0.85 gives a $75 \%$ statistical confidence level for the relationship and an $R^{2}$ value of 0.975 gives a $90 \%$ confidence level. The $R^{2}$ values given in Figures 4 and 5 indicated that all but a few analyte yields were well correlated either with "tar" or with CO yields. The lowest $R^{2}$ values were observed for nitrosamines, quinoline, alkyl phenols and some trace metals.

\section{CONCLUSIONS}

All laboratories used methods they considered most suitable at the time of study. No analytes had statistically lower within-laboratory measurement variability than "tar", and $70 \%$ of the other analytes had statistically higher within-laboratory measurement variability.

All laboratories ranked the products in the same order for all analytes (except some metals) but there was as much as 10fold difference in measured values between laboratories. The mean variation between highest and lowest yield measurements was $80 \%$ even after 3 values in excess of 8 -fold were excluded. This must be taken into account when interpreting data. Data from certain laboratories may appear precise with low variability within a data set, but absolute yields may be quite different to those found at other laboratories.

Given the lack of standardised methods, it is not currently possible to make meaningful comparisons between such data from several sources given the degree of inter-laboratory variability displayed in this study. Indeed, calculation of yields from benchmarking studies may prove no less reliable than the current data.

\section{REFERENCES}

1. Borgerding, M.F., J.A. Bodnar and D.E. Wingate (compilers): The 1999 Massachusetts Benchmark Study. Final Report; A research study conducted after consultation with the Massachusetts Department of Public Health (MDPH), presented to the MDPH on July 24, 2000.

2. Borgerding, M.F., N. Cohen, S.R. Massey, and D.R.E. Thomas, in consultation with M.J. Kaiserman and W S. Rickert: The 1999 Canadian Benchmark Study; provided to Health Canada on May 24, 2000.

3. Hsu, F., J.F. Whidby, B.D. McVeety, N.H. Moore, R.B. Westerberg, H.T. Karnes and S. Bolton: Inter-laboratory analytical methods comparison; 53 ${ }^{\text {rd }}$ Tobacco Science Research Conference, Montreal, Program Booklet and Abstracts, Paper No. 33, 1999. 


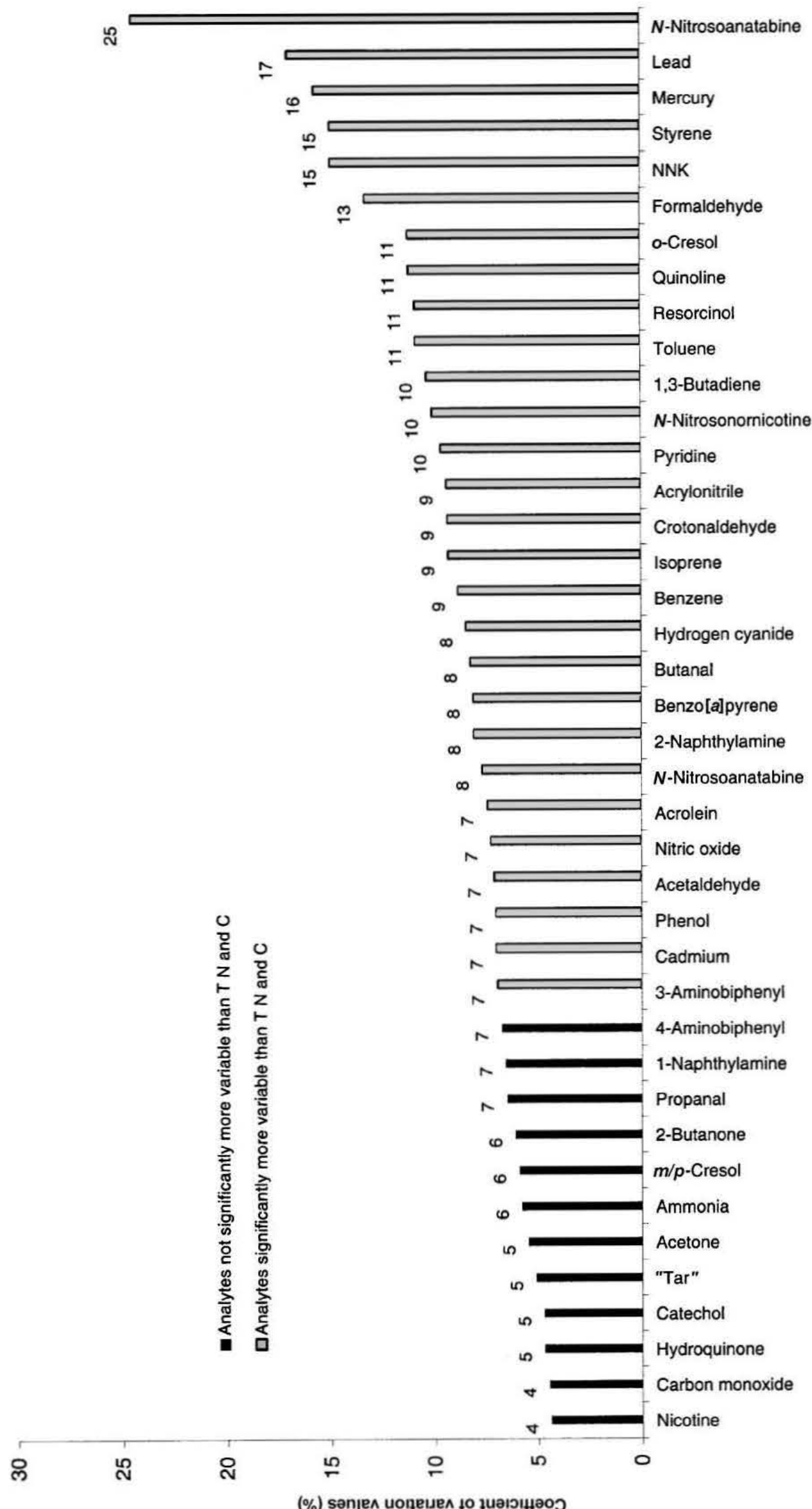

Figure 1. Repeatability (within laboratory variability) 


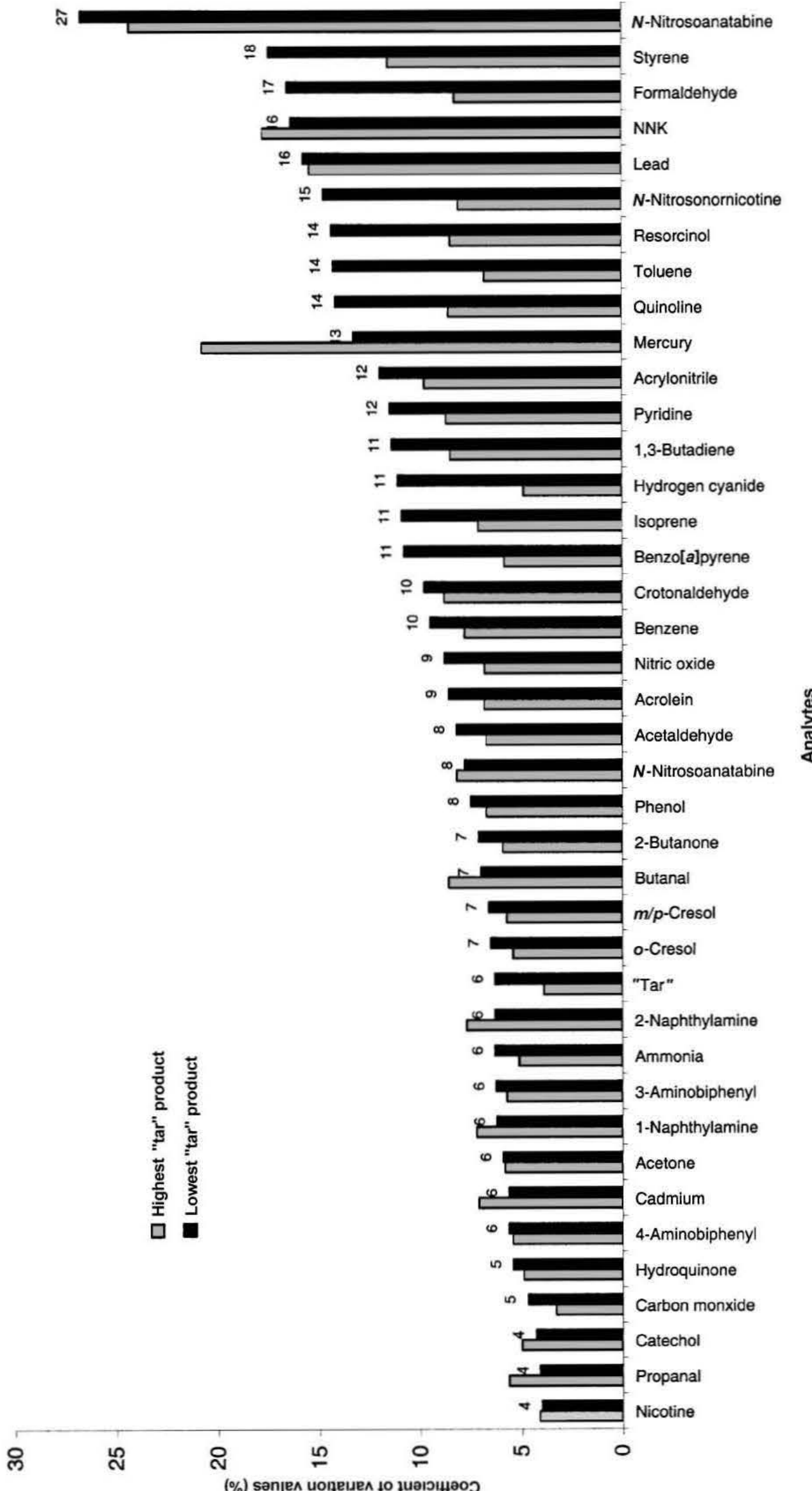

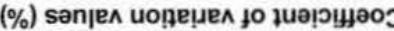

Figure 2. Repeatability (within laboratory variability). Differences between the highest and lowest "tar" products 


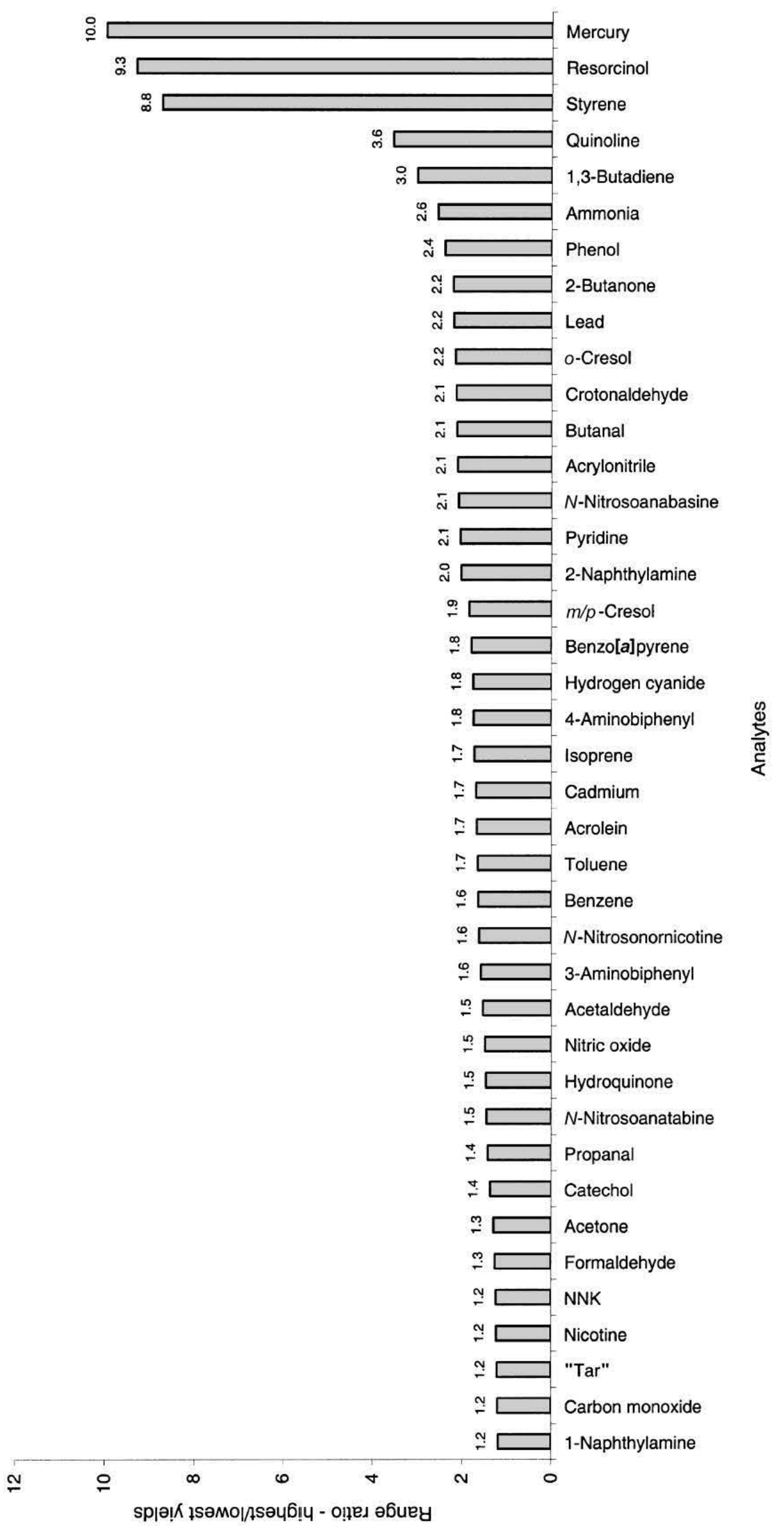

Figure 3. Reproducibility (between laboratory variability). Range ratios of highest to lowest results from laboratories 


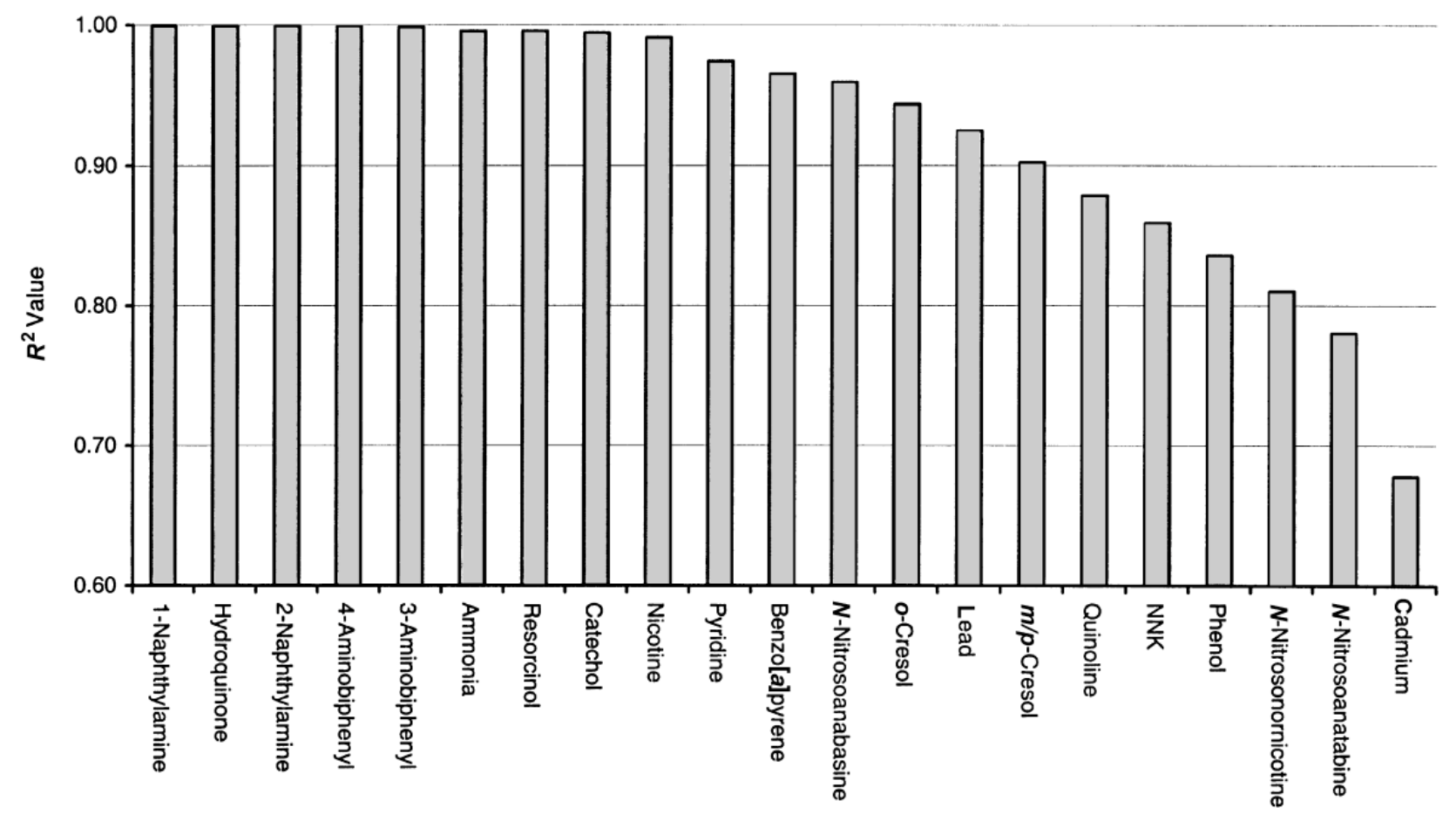

Figure 4. Correlation of analytes with "tar"

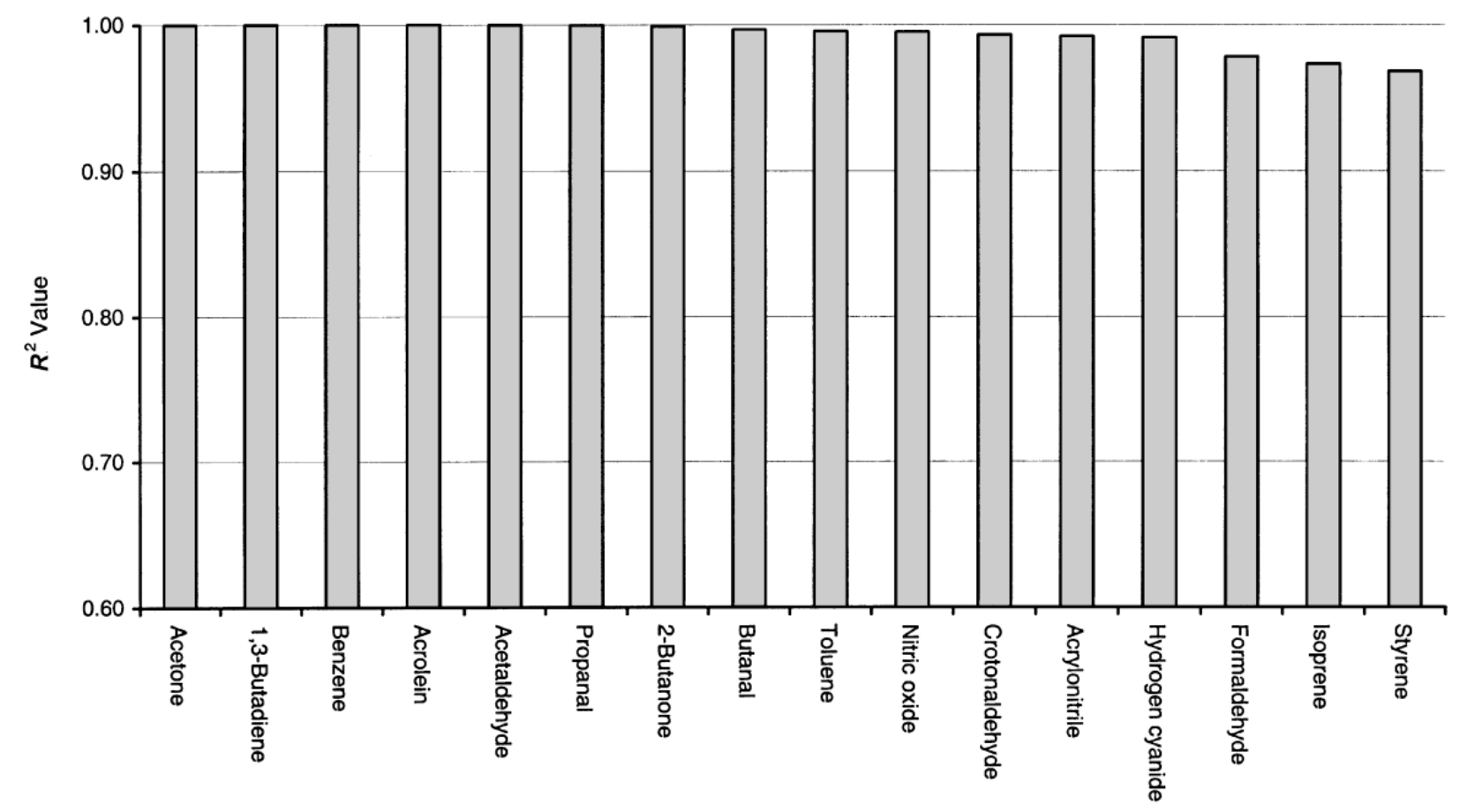

Figure 5. Correlation of analytes with CO

4. ISO 3402: Tobacco and tobacco products - Atmosphere for conditioning and testing; International Organisation for Standardisation, Geneva, 1999.

5. ISO 3308: Routine analytical smoking machine Definition and standard conditions; International Organisation for Standardisation, Geneva, 2000.

6. ISO 5725-2: Accuracy (trueness and precision) of measurement methods and results. Part 2. Basic methods for the determination of repeatability and reproducibility of a standard measurement method; International Or- ganisation for Standardisation, Geneva, 1994.

7. ISO 8243: Sampling tobacco and tobacco products. Part 3. Methods of sampling cigarettes; International Organisation for Standardisation, Geneva, 1991.

Address for correspondence:

Steve Purkis

Imperial Tobacco Limited

P.O. Box 525, Southville

Bristol BS99 1TZ, UK. 